\title{
Bone morphogenetic protein signaling promotes morphogenesis of blood vessels, wound epidermis, and actinotrichia during fin regeneration in zebrafish
}

\author{
Valentine Thorimbert,* Désirée König, ${ }^{*}$ Jan Marro,* Florence Ruggiero, ${ }^{\dagger}$ \\ and Anna Jaźwińska*,1 \\ *Department of Biology, University of Fribourg, Fribourg, Switzerland; and ${ }^{\dagger}$ Institut de Génomique \\ Fonctionnelle-École Normale Supérieure de Lyon, Lyon, France
}

\begin{abstract}
Zebrafish fin regeneration involves initial formation of the wound epidermis and the blastema, followed by tissue morphogenesis. The mechanisms coordinating differentiation of distinct tissues of the regenerate are poorly understood. Here, we applied pharmacologic and transgenic approaches to address the role of bone morphogenetic protein (BMP) signaling during fin restoration. To map the BMP transcriptional activity, we analyzed the expression of the evolutionarily conserved direct phospho-Smad1 target gene, id1, and its homologs $i d 2 a$ and id3. This analysis revealed the BMP activity in the distal blastema, wound epidermis, osteoblasts, and blood vessels of the regenerate. Blocking the BMP function with a selective chemical inhibitor of BMP type I receptors, DMH1, suppressed $i d 1$ and $i d 3$ expression and arrested regeneration after blastema formation. We identified several previously uncharacterized functions of BMP during fin regeneration. Specifically, BMP signaling is required for remodeling of plexus into structured blood vessels in the rapidly growing regenerate. It organizes the wound epithelium by triggering wnt $5 b$ expression and promoting Collagen XIV-A deposition into the basement membrane. BMP represents the first known signaling that induces actinotrichia formation in the regenerate. Our data reveal a multifaceted role of BMP for coordinated morphogenesis of distinct tissues during regeneration of a complex vertebrate appendage.-Thorimbert, V., König, D., Marro, J., Ruggiero, F., Jaźwińska, A. Bone morphogenetic protein signaling promotes morphogenesis of blood vessels, wound epidermis, and actinotrichia during fin regeneration in zebrafish. FASEB J. 29, 000-000 (2015). www.fasebj.org
\end{abstract}

Key Words: blastemal $\cdot$ wound epithelium $\cdot$ regenerative angiogenesis

In contrast to nonregenerative repair or scarring of an amputated limb in mammals, appendage regeneration in zebrafish and urodele amphibians involves the creation

Abbreviations: Aldh1a2, Aldehyde dehydrogenase 1a2; And1, Actinodin 1; BM, basement membrane; BMP, bone morphogenetic protein; BrdU, 5-bromo-2'-deoxyuridine; ColXIV-A, Collagen XIV-A; dpa, days postamputation; EGFP, enhanced green fluorescent protein; FGF, fibroblast growth factor; GFP, green fluorescent protein; hsp70, heat shock protein 70 ;

(continued on next page) of a blastema underneath the wound epidermis (1-4). Current evidence indicates that the blastema is a heterogeneous population of progenitor cells that arises predominantly by dedifferentiation of stump tissues in the vicinity of the amputation (5-9). After the initial establishment of the proliferative blastema, 2 opposing mechanisms have to be activated in the outgrowth (1): maintenance of progenitor cells at the distal position, and (2) redifferentiation of newly generated cells in the proximal region. The inability to dynamically change the cell differentiation status may explain the lack of limb regeneration in higher vertebrates $(10,11)$. Thus, the mechanisms coordinating cellular dedifferentiation and redifferentiation are of central interest in the field of regenerative biology.

Fin regeneration is regulated by epithelial-mesenchymal interactions that involve various signaling molecules, such as Fibroblast growth factor 20a (Fgf20a), Activin- $\beta A$, Wnt, Insulin-like growth factor 2b (IGF2b), Notch, retinoic acid, and Sonic hedgehog $(s h h)$, which have been recently reviewed (12). Although connective tissue provides the primary source of the new structure, other tissue types, such as blood vessels and the skeleton, are important to rebuild the functional organ. The process of angiogenesis occurs through the vascular endothelial growth factor (VEGF)dependent initial plexus formation followed by progressive blood vessel differentiation $(13,14)$. Bone regeneration requires bone morphogenetic protein (BMP) signaling (15-17). The mechanisms controlling regeneration of the actinotrichia, fin-specific skeletal elements, need to be identified (18). Moreover, the coordination of different tissue regeneration, which results in the reconstruction of the complex original appendage, remains poorly understood.

BMPs represent a subgroup of secreted molecules that belong to the TGF- $\beta$ superfamily (19). They initiate the signaling cascade by assembling a heteromeric complex composed of 2 pairs of receptor serine/threonine kinases, known as the type I and type II receptors. The signal is then transmitted by phosphorylation of $\mathrm{Smad} 1 / 5 / 8$, major downstream effectors of canonical BMP signaling. In the

${ }^{1}$ Correspondence: Department of Biology, University of Fribourg, Chemin du Musée 10, 1700 Fribourg, Switzerland. E-mail: anna.jazwinska@unifr.ch

This article includes supplemental data. 
nucleus, phosphorylated Smad1/5/8, in conjunction with other cofactors, bind to BMP-responsive elements of target genes, such as inhibitor of DNA binding-1 (inhibitor of differentiation-1; $i d 1)$. Although the intracellular BMP signal transduction pathway is relatively simple, it is tremendously complex at the level of the signal initiation, mostly because of multiple ligands and receptors, which display considerable promiscuity in their interactions. Therefore, a simultaneous inhibition of all homologs of type I receptors using a pharmacologic approach may offer a valuable way to uncover a complete range of the BMP functions during fin restoration. In the zebrafish fin, the role of the BMP signaling pathway has been focused on bone regeneration (15-17). Other potential functions of this signaling cascade during fin regeneration have not yet been addressed.

\section{MATERIALS AND METHODS}

\section{Animal procedures}

The following zebrafish strains were used in this study: wild-type $A B$ strain (Oregon); flk1 (fetal liver kinase 1):EGFP (enhanced green fluorescent protein) $(20,21)$; tie 2 (tyrosine kinase with immunoglobulin-like and EGF-like domains 2):EGFP (22); osterix:nlsGFP (nuclear localization signal green fluorescent protein); osteocalcin: GFP (green fluorescent protein); runx2 (Runt-related transcription factor 2):GFP (6); hsp70 (heat shock protein 70):bmp2b; and hsp70:noggin 3 (23). Regeneration studies were performed as described previously at $26.5^{\circ} \mathrm{C}$ (24). Stock solutions of DMH1 (Tocris Bioscience, Bristol, United Kingdom) and PTK787 (Selleckchem, Houston, TX, USA) were dissolved in DMSO at the concentration of $10 \mathrm{mM}$. During treatments, water was changed every $2 \mathrm{~d}$. For proliferation analysis, fish were incubated for $7 \mathrm{~h}$ in fish water containing $50 \mu \mathrm{g} / \mathrm{ml}$ 5-bromo-2'-deoxyuridine (BrdU; Sigma-Aldrich, St. Louis, MO, USA). Heat shock was induced at $37^{\circ} \mathrm{C}$ for $1 \mathrm{~h}$. The cantonal veterinary office of Fribourg approved the experimental research on animals. In vivo imaging was performed with a Leica M205 FA fluorescence stereomicroscope (Leica Microsystems, Renens VD, Switzerland). ImageJ 1.46r software (NIH, Bethesda, $\mathrm{MD}$, USA) was used for measurements of the regenerate area.

\section{Production of Actinodin 1 peptide antibodies}

A peptide, CGQDDHLAYNGDYRKK, which corresponds to the real C terminus of Actinodin 1 (And1) protein with an additional cysteine residue at the amino terminus of the peptide, was synthesized and injected into rabbits to generate polyclonal And1 antibodies used at 1:5000 (Eurogentec, Liège, Belgium).

\section{Histologic analysis and statistics}

The following primary antibodies were used: rabbit $\mathrm{mAb}$ antiphospho-Smad1/5, 1:100 (41D10; Cell Signaling Technology, Danvers, MA, USA); mouse anti-Zns5, 1:500 (Zebrafish Information Network, Eugene, OR, USA); rabbit anti-Tenascin C, 1:500 (USBiological, Hamburg, Germany); mouse anti-Claudin-5, 1:100 (Invitrogen, Life Technologies, Carlsbad, CA, USA); rat anti-BrdU, 1:100 (Abcam Inc., Cambridge, MA, USA); rabbit anti-Aldh1a2 (Aldehyde dehydrogenase 1a2), 1:800 (GeneTex, Irvine, CA, USA); rabbit anti- $\alpha$ Catenin, 1:2000 (Sigma-Aldrich);

(continued from previous page)

$i d$, inhibitor of differentiation (inhibitor of DNA binding); IGF, Insulin-like growth factor; nls, nuclear localization signal; pSmad, phospho-Smad; runx2, Runt-related transcription factor 2; shh, Sonic hedgehog; VEGF, vascular endothelial growth factor chicken anti-GFP, 1:1000 (Aves Labs, Tigard, OR, USA); and rabbit anti-active Caspase-3, 1:10,000 (Abcam Inc.). Guinea pig anti-ColXIV-A (Collagen XIV-A), 1:1000, was a kind gift from F.R. The secondary antibodies used (at 1:500) were Alexa conjugated (Jackson ImmunoResearch Laboratories, West Grove, PA, USA).

Fins were fixed in $4 \%$ paraformaldehyde and immunostained as previously described (25). The specimens were analyzed by confocal microscopy (Leica TCS-SP5). To enhance the visibility of the blue fluorescent color on the final figures, we modified the color profile with Adobe Photoshop (San Jose, CA, USA) using CMYK conversion. For quantification of BrdU-positive nuclei in the blastema at $3 \mathrm{~d}$ postamputation (dpa), we calculated the proportion of immunostained cells per total number of DAPI-stained nuclei using ImageJ 1.46r software. Error bars correspond to SEM. Significance of differences was calculated using Student's $t$ test.

\section{In situ hybridization}

In situ hybridization on cryosections was performed as previously described (24). Primers for PCR amplification of genes to generate antisense probes were the following: $i d 1$, forward 5 '-CAGCAAAGTTGGAGGAGAGG-3' and reverse 5'-AGCCGTTCTCCACAGAGATGT-3'; $i d 2 a$, forward 5'-GCAATAAGCCCAGTGAGGTC-3' and reverse 5'-GTTATCGCCGAATTGGAGTC-3'; $i d 3$, forward 5' ${ }^{\prime}$ TGTGCTGTATCTCGGAGCAG-3' and reverse $5^{\prime}$ TGGCACATAGCTCCATCTTC-3'; $b m p 7 a$, forward 5'-GCGGGAAATCCTCTCTATCC-3' and reverse 5' ${ }^{\prime}$-TCAAAGGGAACACACATTCG-3'; $b m p 2 b$, forward 5'-CTCTCACGGTGCTGTTGCTC$3^{\prime}$ and reverse $5^{\prime}$-TCTCGGTTCGAATGCAAGAC-3'; alk1, forward 5'-CTGGTGTTGTTGGTGATTGC-3' and reverse 5'-AGGGCACCATGTCAAAGAAC-3'; alk2, forward 5'-CATTGACTGCATGTGTGTCG-3' and reverse 5'-TCAGGTCTCTGTGAGCGATG-3'; bmprla, forward 5'-CATCACTGTGGTTTTGACTGG-3' and reverse 5' ${ }^{\prime}$-CTGGGATAAAGGCCTCGTCT-3'; bmprlb, forward 5'-TGTGCTGCTCATTGGGCTTC-3' and reverse 5'-TTCTCCAGCAGGAATGAAGC-3'; $m s x b$, forward 5'-GAGAATGGGACATGGTCAGG-3' and reverse 5'GCGGTTCCTCAGGATAATAAC-3'; aldh1a2, forward 5'-GGCTGATCTGGTGGAGAGAG-3' and reverse 5'-TGAATCCTCCGAAAGGACAC-3'; lef1, forward 5'-CAGTCACGACGCAGCTAGAC-3' and reverse 5'-CTCTGGCGTGTACCTGAAGC-3'; sh $a$, forward 5'-GACACCTCTCGCCTACAAGC-3' and reverse 5'-GGAACAGGAATGATGACACG-3'; and wmt5b, forward 5'-ACTCGTGGTGGTCATTAGCG-3' and reverse 5'-TACCCTCCGAGGTCTTGTTG-3'.

The reverse primers were synthesized with addition of the promoter for T3 polymerase. To assess $i d$ gene expression in endothelial cells, the sections of tie2:EGFP fish were immunostained against GFP after completion of the in situ hybridization protocol.

\section{Quantitative real-time PCR analysis}

cDNA synthesis and quantitative RT-PCRs were performed as previously described (25). The values and expression ratios were normalized to $\beta$-actin. The following primers were used: $\beta$-actin 2 , forward 5'-TTGGCAATGAGAGGTTCAGG-3' and reverse $5^{\prime}$ TGGAGTTGAAGGTGGTCTCG-3'; $i d 1$, forward 5'-AATGCAAGATCCCGCTGCTG-3' and reverse 5'-CTTGTTGGTCGGTAGCGTGG-3'; and and1, forward 5'-CCGAACCCGTCAGTATTTCC-3' and reverse 5'-GCGTCATATTGCTGCTGGTC-3'

The normalized gene expression values were calculated with the Pfaffl method.

\section{RESULTS}

\section{BMP signaling is required for the progression of regeneration}

The expression of several BMP ligands has been previously analyzed in the fin regenerate. Specifically, $b m p 2 b$ is expressed 
in osteoblasts, $b m p 4$ in the distal blastema, whereas $b m p 6$ is present in osteoblasts, blastema, and wound epidermis (15, $16,26)$. Here, we expanded this analysis by the identification of another BMP ligand, $b m p 7 a$, which we detected in the distal blastema and epidermis of the fin at $3 \mathrm{dpa}$ (Supplemental Fig. S1A). Thus, the expression of BMP ligands is complex and partially overlapping. To reveal tissues that are responsive to the BMP signals, we analyzed the expression of all homologs of BMP type I receptors, namely alk1 (acurl1), alk2 (alk8and acvr1), alk3 (bmprla), and alk6 (bmpr1b). In situ hybridization of fins at 3 dpa demonstrated that nearly all of the fin tissues, including wound epidermis, blastema, osteoblasts, and blood vessels, express at least one BMP receptor homolog (Supplemental Fig. S $1 B-E$ ). A partially overlapping expression pattern of some of the ligands and receptors may indicate a potential functional redundancy, which has previously been documented in various developmental contexts (19). The broad distribution of the signaling components suggests that the BMP pathway could be involved in multiple regenerative processes. Interestingly, $h s p 70$ promoter-driven overexpression of $b m p 2 b$ or its antagonist noggin 3 in transgenic fish resulted in teratogenic effects during fin regeneration, indicating limitations of this approach (Supplemental Fig. S2).

To study this multiligand/receptor signaling pathway in the regenerating fin, we applied a pharmacologic approach to globally inhibit all related BMP type I receptors using DMH1 (27). This inhibitor has been shown to selectively suppress phosphorylation of Smad1/5/8 at the concentration of $10 \mu \mathrm{M}$ without disrupting other signaling pathways, such as TGF- $\beta$ /Activin/pSmad2, kdr/VEGF/ p-Flk1, and p38/p-MAPK (28). To characterize the role of BMP signaling during fin regeneration, fish were preincubated with $10 \mu \mathrm{M}$ DMH1 for $1 \mathrm{~d}$, and the treatment was continued during the regenerative process for $10 \mathrm{~d}$. Until $3 \mathrm{dpa}$, the outgrowth size of the DMH1-treated fins was only slightly smaller than that of the control fins $($ Fig. $1 A-D)$. However, starting from this stage, the elongation of the regenerate was markedly impaired, indicating an arrest of regeneration after blastema formation (Fig. $1 E, F)$. We concluded that BMP signaling is not essential for the initiation of the regenerative program but for the subsequent progression of regeneration. To test the requirement of BMP specifically during the advanced regeneration phase, we performed the DMH1 treatment starting from $3 \mathrm{dpa}$ until $6 \mathrm{dpa}$. The inhibition of BMP signaling during this time window reduced fin elongation, supporting the notion that BMP is required to promote regeneration (Supplemental Fig. S3).

To analyze cell proliferation within the regenerate after $3 \mathrm{~d}$ of DMH1 treatment, we performed a BrdU incorporation assay (Fig. 1G). We found a decrease of proliferating epithelial cells despite the thickened appearance of the wound epidermis, suggesting a role of BMP signaling in the organization of this tissue (Fig. $1 H, K, L$ ). However, proliferation of blastema cells was only moderately reduced after $3 \mathrm{~d}$ of treatment and remained unchanged after $1 \mathrm{~d}$ of treatment, demonstrating that BMP does not act as direct mitogen in the outgrowth (Fig. $1 H, I, j$; data not shown). Taken together, the formation of the blastemal outgrowth and the mild effect on mesenchymal cell proliferation suggest that the DMH1 treatment, even for $3 \mathrm{~d}$, does not affect mitogenic pathways, such as FGF, Activin$\beta A$, IGF, and retinoic acid, all of which are known to be required for initiation of the regeneration program (12).

In addition to the decreased size of the regenerate, we observed 2 morphologic phenotypes, namely defective bones and conspicuous blood clots at the distal margin (Fig. $1 D, F)$. To test whether the severity of the phenotype correlates with the concentration of the drug, we repeated the live-imaging analysis using different DMH1 concentrations. The treatment with $1 \mu \mathrm{M}$ DMH1 partially suppressed regeneration as compared with $10 \mu \mathrm{M}$, indicating a dosedependent severity of the phenotype (Supplemental Fig. S4). Treatment with $1 \mu \mathrm{M}$ DMH1 did not affect cell proliferation at $3 \mathrm{dpa}$ (data not shown). The concentration of $20 \mu \mathrm{M} \mathrm{DMH} 1$ induced a severe reduction of the outgrowth, hemorrhages, and abnormal rays, which resembled the effects caused by $10 \mu \mathrm{M}$ DMH1 (Supplemental Fig. S4). Thus, the concentration of $10 \mu \mathrm{M}$ DHM1 is appropriate for phenotypic analysis of fin regeneration in adult fish.

\section{Mapping of the BMP activity using endogenous pSmad1/5/8 target gene $i d 1$}

To identify tissues that are dependent on BMP signaling, we visualized downstream activation of the BMP signaling pathway. A reliable readout of BMP signaling is the visualization of nuclear pSmad1/5/8. At 1 and $2 \mathrm{dpa}$, monoclonal rabbit antibody against pSmad $1 / 5 / 8$ detected the activity of BMP signaling in the basal layer of wound epidermis, osteoblasts, and blood vessels of transgenic fish flk1:GFP at the proximity of the amputation plane and in the outgrowth (Fig. $1 M-P$ ). At $3 \mathrm{dpa}, \mathrm{pSmad} 1 / 5 / 8$ were additionally observed in the distal-most blastema (Fig. $1 Q$, $R)$. The treatment with DMH1 for $3 \mathrm{~d}$ or only for $1 \mathrm{~d}$ starting at 2 dpa suppressed pSmad1/5/8 immunoreactivity, which confirms the efficiency of our pharmacologic approach (Fig. $1 S$ and Supplemental Fig. S5C,D).

To visualize the transcriptional activity of BMP signaling, we analyzed the expression of the evolutionarily conserved immediate pSmad1/5/8 target gene $i d 1$ (29). The expression pattern of $i d 1$ correlated with the distribution of pSmad1/5/8 at different time points of regeneration, reflecting the BMP signaling activity (Fig. $2 A$ and Supplemental Fig. S5E, $F$ ). Given that $i d$ genes have not yet been analyzed in the zebrafish fin, we visualized the expression of other $i d$-related genes, namely $i d 3$ and $i d 2 a$, during the regenerative outgrowth phase. At $3 \mathrm{dpa}, i d 1$ and $i d 3$ transcripts were detected in a nearly overlapping pattern of the outgrowth, specifically in the distal-most blastema, osteoblasts, wound epidermis, and blood vessels, which were visualized by endothelial EGFP expression in transgenic fish tie2:EGFP (Fig. 2A, $C-H$ and Supplemental Fig. S6). Similar expression patterns, with the exception of vasculature, were also detected with the probe against $i d 2 a$ mRNA (Fig. 2Jand Supplemental Fig. S6). The sites of $i d 1$ transcription correlated with the $\mathrm{pSmad} 1 / 5 / 8$ distribution pattern (compared with Fig. 1Q). Importantly, the DHM1 treatment completely suppressed $i d 1$ and $i d 3$ expression, but it did not markedly affect $i d 2 a$ expression (Fig. 2B, $I, K$ ). Moreover, a pulse treatment with DMH1 for $1 \mathrm{~d}$ starting at 2 dpa was sufficient to down-regulate $i d 1$ expression (Supplemental Fig. S5A, $B, G-J$ ). Thus, we identified $i d 1$ as a BMP target gene in the fin. 

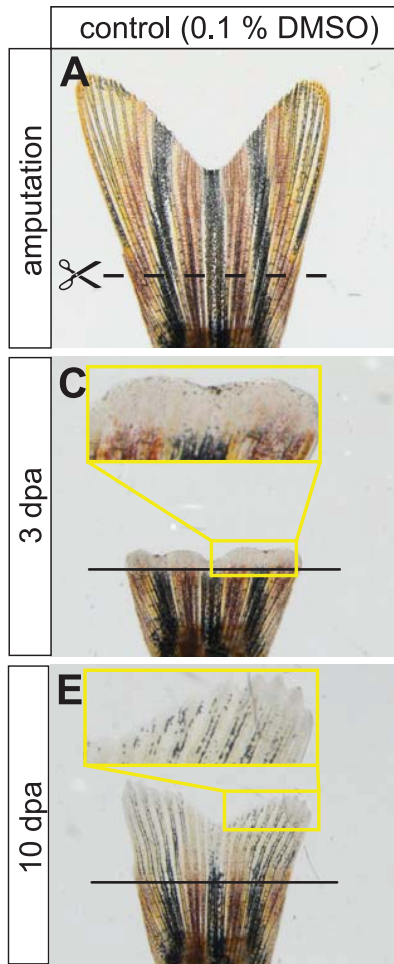

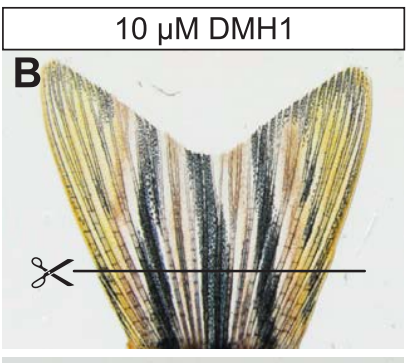

D

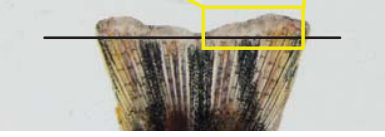

$\mathbf{F}$

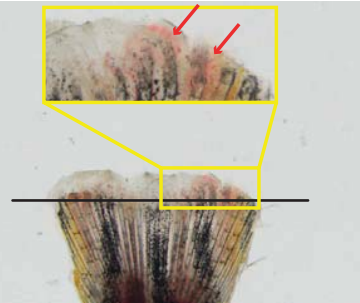

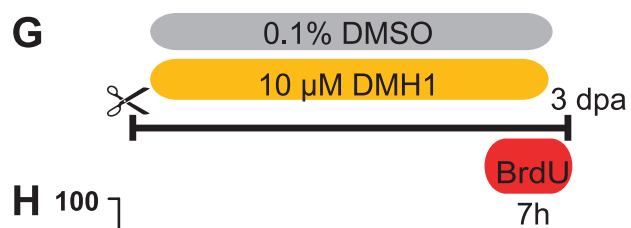

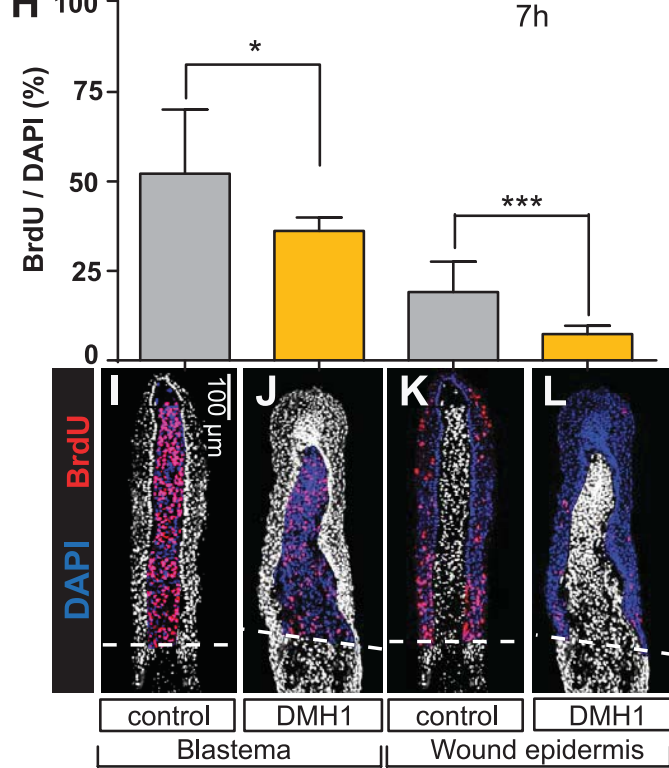

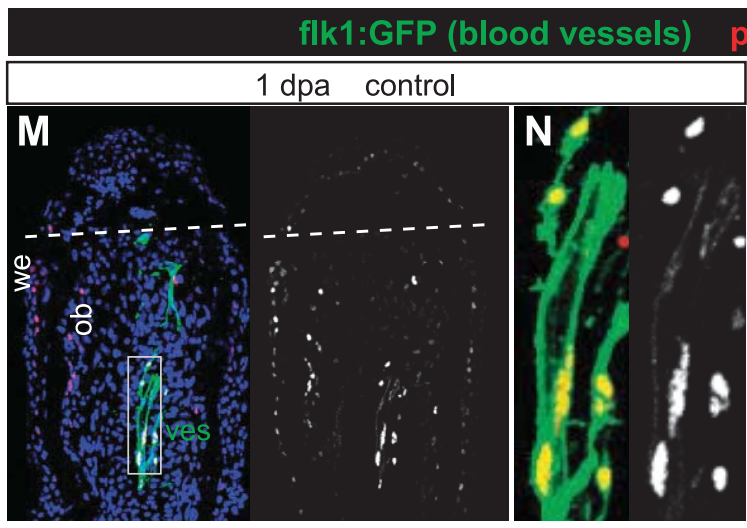

pSmad1/5/8 (pSmad1/5/8) DAPI
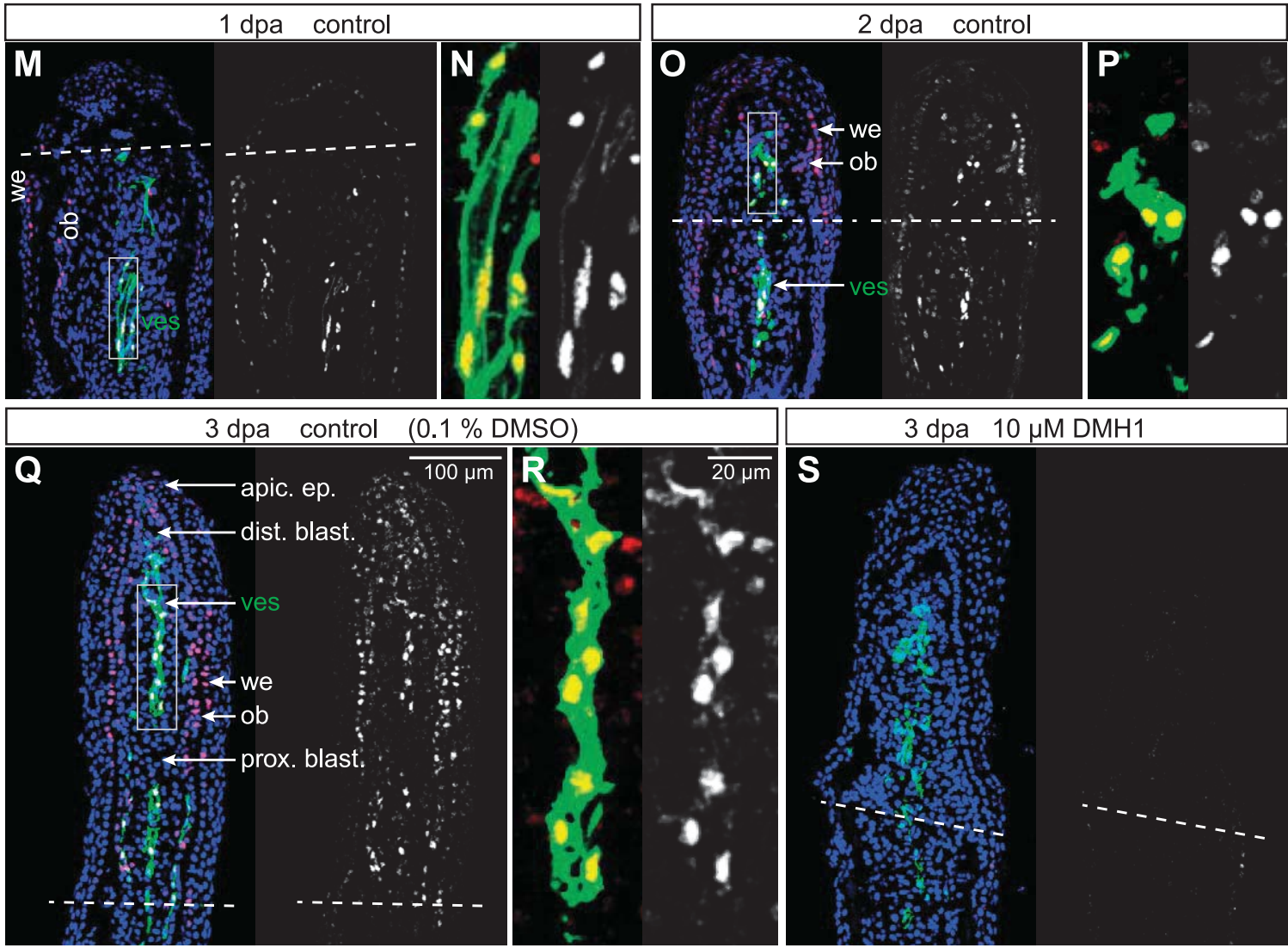

Figure 1. DMH1-mediated inhibition of BMP signaling arrests fin regeneration during the early outgrowth phase. $A-F)$ Live imaging of whole fins at different time points of regeneration. $A$ and $B$ ) Original fins before amputation (dashed lines). $C$ and $D$ ) At $3 \mathrm{dpa}$, an outgrowth of new tissue emerges above the amputation plane. $E$ and $F$ ) At $10 \mathrm{dpa}$, control fins display advanced regeneration, whereas DMH1-treated fins failed to progress this process. $F$ ) The outgrowth contains hemorrhages (red arrows) $(n=5) . G$ and $H)$ The BrdU assay at $3 \mathrm{dpa} . G)$ Experimental design. $H$ ) Quantification of BrdU-positive nuclei in the blastema (continued on next page) 
BMP is not essential for the initiation of the regeneration program in mesenchyme and bones

The normal initiation of the outgrowth formation indicates that BMP signaling is not essential for blastema formation. To verify this hypothesis, we analyzed the expression of several known blastema markers, Tenascin C, $m s x b$, and aldh1a2 (raldh2), all of which are induced in the mesenchyme of the outgrowth $(25,30,31)$. At 3 dpa, the DMH1 treatment did not suppress the expression of these genes (Fig. 3A-H). We concluded that BMP signaling is not essential for the activation of the regeneration program in the mesenchyme.

Previous studies demonstrated $b m p 2 b$ expression in regenerating osteoblasts and the requirement of BMP signaling during bone regeneration (15-17, 26). At $3 \mathrm{dpa}$, the treatment with DMH1 did not affect the induction of $b m p 2 b$ transcription in osteoblasts at the amputation site, but it impaired their migration into the outgrowth (Fig. 3I, J). The differentiation status of osteogenic cells can be elegantly monitored using 3 transgenic fish lines: osteoprogenitors are marked by the runx2:GFP transgenic reporter, committed osteoblasts by osterix:nlsGFP, and mature osteoblasts by osteocalcin:GFP (6). We treated these 3 transgenic fish lines with DMH1 and performed live imaging at $6 \mathrm{dpa}$. In agreement with previous studies (17), BMP signaling was not essential for osteoblast dedifferentiation but redifferentiation, as visualized by persistent runx2:GFP expression and impaired osterix:GFP and osteocalcin:GFP activation (Fig. 3M-R). Importantly, the phenotype was not associated with apoptosis, as shown by active Caspase- 3 staining of fin sections (Fig. $3 K, L$ ).

\section{BMP signaling is required for plexus remodeling into structured blood vessels in the regenerate}

The presence of pSmad1/5/8 in endothelial cells at the site of injury implicates a direct activity of BMP signaling during blood vessel regeneration (Fig. $1 M-R$ ). To identify the function of BMP during regenerative angiogenesis, we analyzed the fin vasculature of $f l k 1$ :GFP transgenic fish after DMH1 treatment. At $3 \mathrm{dpa}$, the initial revascularization of the regenerate was comparable between control and DMH1-treated fins (Fig. 4A, B). However, when the same fish were analyzed at $10 \mathrm{dpa}$, the progression of angiogenesis was impaired (Fig. $4 C, D$ ).

The initial recruitment of blood vessels into the outgrowth suggests that inhibition of BMP does not impair the ability of endothelial cells to recognize angiogenic VEGF signaling. It is known that blocking the VEGF receptor using PTK787 during fin regeneration completely prevents angiogenesis, restricting the size of the outgrowth due to insufficient blood supply (14). We asked the question whether a similar correlation between the deficient vascularization and the regeneration arrest also occurs in DMH1-treated fins. To this aim, we compared the total outgrowth length and its nonvascularized part between DMH1- and PTK787-treated fins (Fig. 4A-D and Supplemental Fig. S7A). Although the total size of the regenerate at 10 dpa was comparable between both treatments, the inhibition of the VEGF receptor allowed for the formation of twice-longer nonvascularized tissue (Supplemental Fig. S7B). This suggests that the blockage of regeneration after BMP inhibition cannot be explained exclusively by the impaired progression of angiogenesis.

To characterize the organization of the regenerating vasculature, we performed live imaging of transgenic fish tie2:EGFP. At 3 dpa, control and DMH1-treated fins elaborated the vascular plexus in each ray blastema (Fig. $4 E, F$ ). The distribution of vascular branches was denser in the DMH1-treated fins than in control, which could be explained as a secondary effect due to the arrest of regeneration at this stage. At $6 \mathrm{dpa}$, control fins displayed a gradual remodeling of the plexus into a network of refined branches with the stereotypic vein-artery-vein architecture of the ray (Fig. $4 G$ ). By contrast, the inhibition of BMP signaling blocked the transition from the plexus into the mature vasculature pattern (Fig. $4 H$ ). Specifically, the regenerates contained unstructured dilated vessels with little spacing. Fragments of the endothelium were frequently observed in the distal fin margin, suggesting a disrupted integrity of the blood vessels (Fig. $4 H$ ). This suggests that BMP signaling is required for remodeling of the vascular plexus, the maturation of blood vessels, and the maintenance of their integrity. To verify this conclusion, we blocked BMP signaling for $2 \mathrm{~d}$ starting at $6 \mathrm{dpa}$, after the initial plexus remodeling. This pulse DMH1 treatment transformed preexisting veins and arteries of the blastema into a bunch of dilated collapsed blood vessels with a disrupted integrity (Supplemental Fig. S7C, D). Thus, BMP signaling is required to maintain the structure of the regenerating blood vessels.

To further examine the morphology of blood vessels, we analyzed transversal sections of the blastema in flk1:GFP fish at $6 \mathrm{dpa}$. Control fins displayed narrow capillaries with a regular distribution along the midline of the rays between the concave hemirays of osteoblasts that were visualized with the Zns5 antibody (Fig. 4I). We found that the osteoblast-deficient part of the blastema in DMH1-treated fins contained misshaped capillaries, supporting our liveimaging observations (Fig. $4 J$ ).

To test if the dense plexus of DMH1-treated fins might be due to overproliferation of endothelial cells, we performed a BrdU incorporation assay. We exposed flk1:GFP transgenic fish to DMH1 for only $1 \mathrm{~d}$ starting at $2.5 \mathrm{dpa}$ and additionally to BrdU for $7 \mathrm{~h}$ before fin fixation. Although

and wound epidermis of the fin outgrowth $(n=10) . * P<0.05$; ***P<0.0005. Error bars represent the SEM. $I-L)$ Representative images of longitudinal fin sections for quantification of proliferating cells. The analyzed tissue compartments are shown in color, whereas the remaining part is in white (DAPI). $M-R$ ) Immunofluorescence staining of control fin sections of $f l k 1$ :GFP transgenic fish using monoclonal rabbit antibody detects pSmad1/5/8 (red in the left panels; white in the right panels) in apical epidermis (apic. ep.), distal blastema (dist. blast.), basal layer of wound epidermis (we), osteoblasts (ob), and blood vessels (ves). No pSmad1/5/8 immunofluorescence was observed in the proximal blastema (prox. blast.) $(n=4) . N, P$, and $R$ ) Higher magnifications of the blood vessel framed in the left panels demonstrate pSmad1/5/8 in endothelial cells of the outgrowth. $S)$ DMH1 treatment completely abolishes pSmad1/5/8 $(n=4)$. 


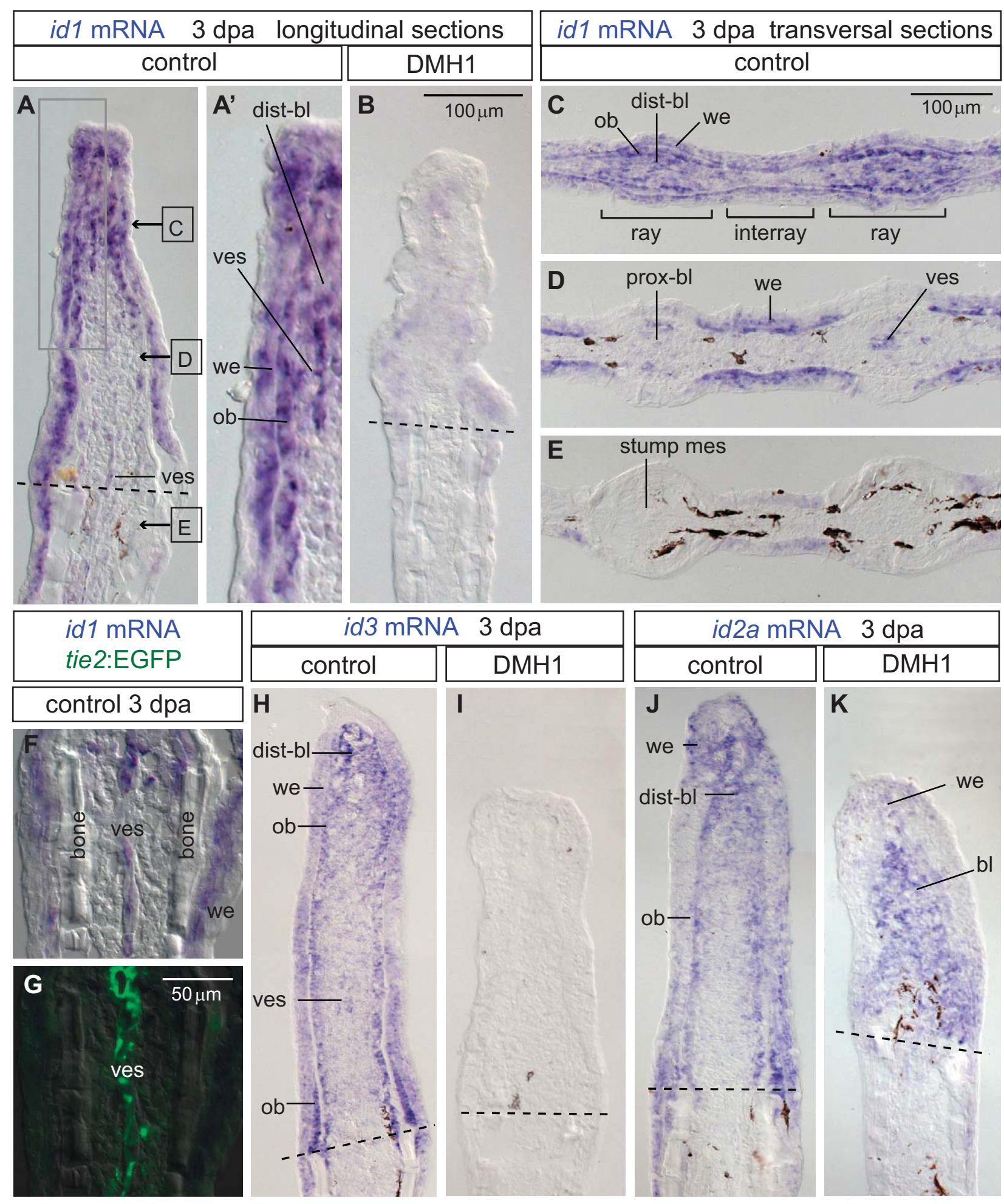

Figure 2. Expression of $i d 1$ and $i d 3$ genes reflects the transcriptional activity of BMP signaling in multiple tissues of the fin regenerate. $A-E)$ In situ hybridization of longitudinal $(A)$ and transversal $(C-E)$ fin sections at 3 dpa shows $i d 1$ expression in the distal blastema (dist-bl), blood vessel (ves), wound epidermis (we), and osteoblasts (ob). id1 mRNA is absent in the proximal blastema (prox-bl) and stump mesenchyme (stump mes). B) No id1 expression is detected after DMH1 treatment $(n=4)$. F and $G)$ Section of fin from tie2:EGFP transgenic fish demonstrates coexpression of $i d 1$ and GFP in the blood vessel at the fin outgrowth $(n=4)$. $H$ and $I) i d 3$ mRNA is detected in the similar pattern to $i d 1 \mathrm{in}$ control fins $(H)$, and it is completely suppressed by DMH1 treatment $(I)(n=4)$. $J$ and $K) i d 2 a$ expression pattern is reminiscent of $i d 1 / i d 3$ in control fins, except the blood vessels $(J)$. $K)$ DMH1 treatment does not suppress $i d 2 a$ transcription $(n=4)$. 

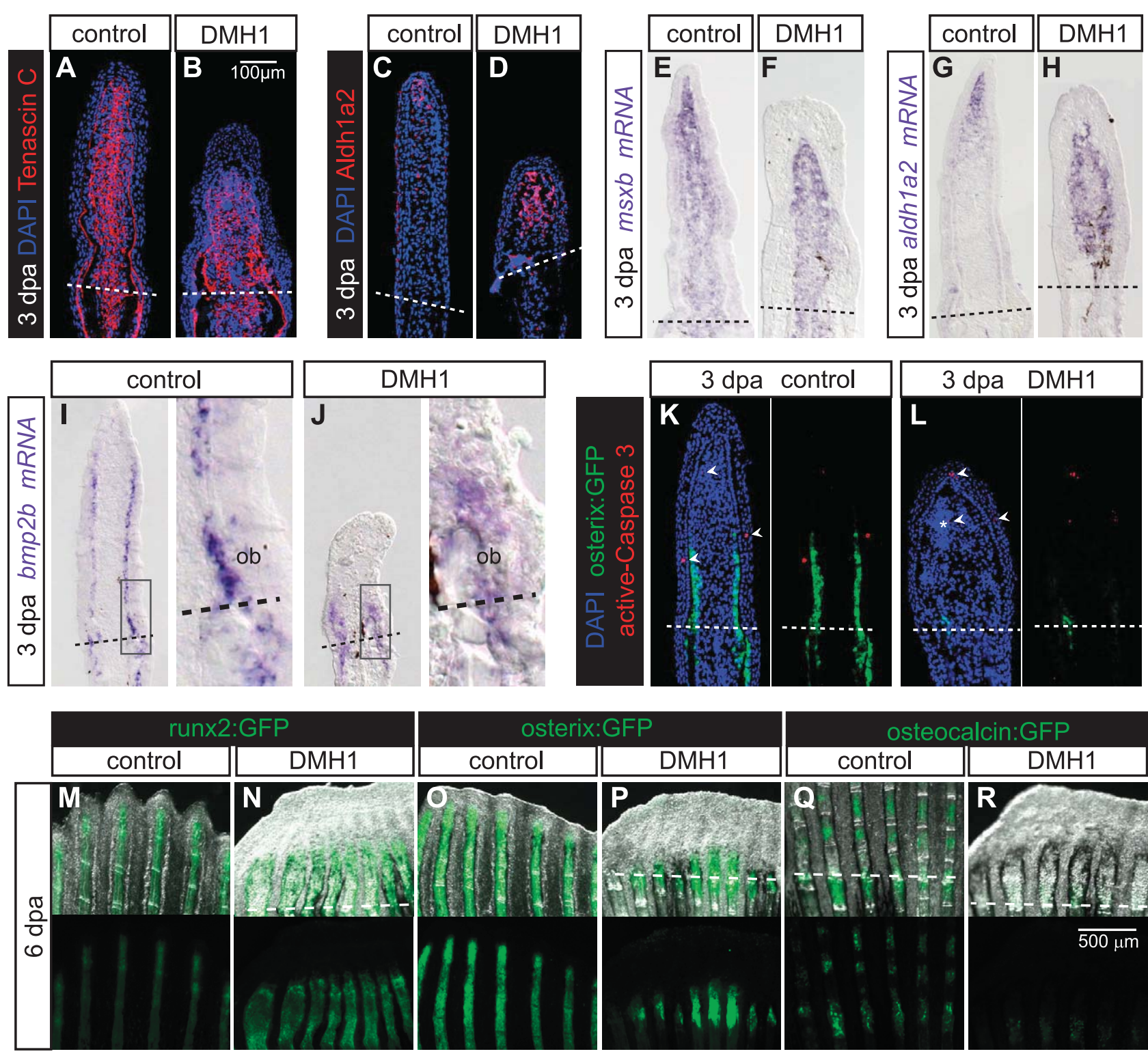

Figure 3. BMP signaling is not required for initial blastema formation but for bone differentiation. $A-D$ ) Immunofluorescence staining of longitudinal fin sections at $3 \mathrm{dpa}$. $A$ and $B$ ) Tenascin $\mathrm{C}$ is expressed in the entire blastema in control and DMH1treated fins. $C$ and $D$ ) Aldhla2 is restricted to the distal blastema in control, but it expands in the entire blastema after DMH1 treatment $(n>4) . E-J)$ In situ hybridization of fin sections at $3 \mathrm{dpa} . E$ and $F$ ) The whole blastema marker $m s x b$ is not affected by DMH1 treatment. $G$ and $H$ ) aldh1a2 mRNA is confined to the distal outgrowth of control. It is detected in the blastema of DMH1treated fins. $I$ and $J) b m p 2 b$ is expressed in regenerating osteoblasts in control. It is also induced in DMH1-treated fins in the vicinity of amputation, but osteoblast migration is impaired $(n>4)$. $K$ and $L)$ At 3 dpa, osterix:nlsGFP is suppressed in DMH1treated fins compared to control. No change in apoptotic cells (anti-active Caspase-3, red, white arrowheads) is observed between the samples $(n=5) . M-R$ ) Live imaging of transgenic fish demarcating distinct differentiation levels of osteoblasts at 6 dpa. $M-P$ ) In control fins, the distal half of the outgrowth reduces expression of runx2:GFP, a marker of preosteoblasts, as the cells enter differentiation and activation of osterix:GFP, a marker of committed osteoblasts. DMH1 treatment prevents this transition and maintains strong runx2:GFP instead of osterix:GFP. $Q$ and $R$ ) The proximal part of the outgrowth initiates expression of osteocalcin: GFP in control, indicating osteoblast maturation. Activation of osteocalcin:GFP does not take place in DMH1-treated fins $(n=4)$.

BrdU abundantly labeled the outgrowth, we found a small decrease in endothelial cell proliferation (Supplemental Fig. S7E). Thus, the excessive vasculature in the plexus of DMH1-treated fins is not likely to derive from enhanced proliferation of endothelial cells but probably from malformation of the blood vessels. Taken together, BMP signaling regulates the structure of the vascular network during growth of the regenerate.
BMP signaling regulates the basal layer of the wound epidermis

The wound epidermis consists of 3-4 layers of epidermal cells, among which the basal layer displays a distinctive regular organization $(24,32,33)$. We found that the DMH1 treatment resulted in approximately twice-thicker wound epidermis, which contained 6-10 cell layers (Figs. 4I, Jand 

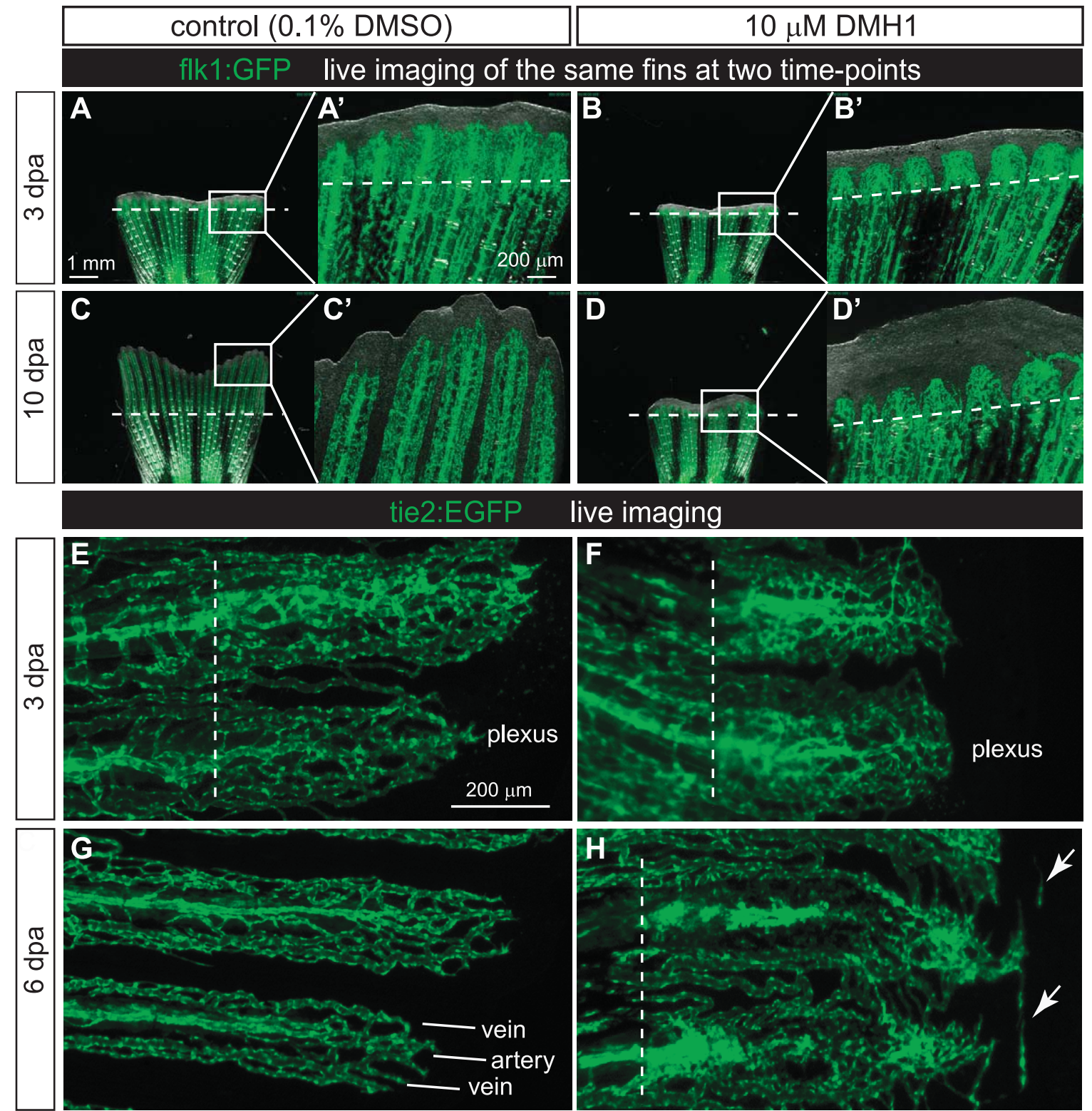

\section{flk1:GFP Zns5 DAP| flk1:GFP transversal sections of the blastema}
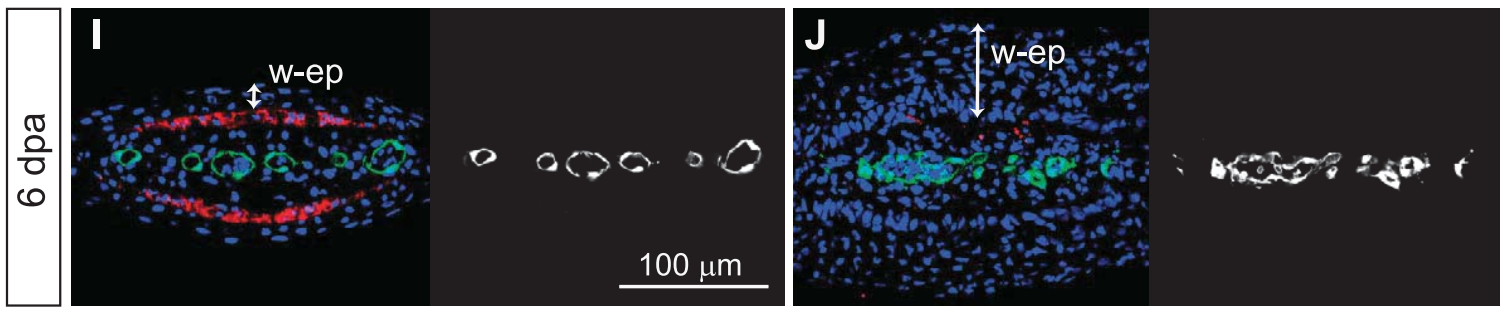

Figure 4. Exposure to DMH1 impairs plexus remodeling into mature blood vessels. $A-D)$ Live imaging of $f k 1$ :GFP transgenic fish at 3 and $10 \mathrm{dpa}$. At $3 \mathrm{dpa}$, normal vascularization is observed in control $(A)$ and DMH1-treated fish $(B)$. At 10 dpa, the outgrowth of control fins $(C)$ is vascularized nearly until its margin, whereas DMH1-treated fins $(D)$ failed to progress angiogenesis. A distal portion of the outgrowth remains nonvascularized. $A^{\prime}, B^{\prime}, C^{\prime}$, and $\left.D^{\prime}\right)$ Higher magnifications of the framed area in the corresponding images $(n=5)$. $\left.E-H\right)$ Live images of fin regenerate margin from tie2:EGFP transgenic fish. $E$ ) In control at $3 \mathrm{dpa}$, regeneration of blood vessels involves the formation of plexus. G) At 6 dpa, the plexus undergoes remodeling toward the final stereotypic vascular pattern, with a central artery in the middle of the ray and 2 lateral veins. F) At 3 dpa, DMH1-treated fins form the plexus in the outgrowth. $H$ ) At 6 dpa, the DMH1 treatment prevents plexus remodeling, resulting in a dense network of disorganized blood vessels. Arrows indicate detached fragments of the endothelium. The regenerates are to the right from the amputation planes (dashed lines) $(n=5) . I$ and $J$ ) Transversal sections of regenerate from flk1:GFP transgenic fish at $6 \mathrm{dpa}$. Control fins $(I)$ display normal bone regeneration visualized by Zns5 antibody staining (red) and an array of capillaries (green) along the midline of the fin. The vasculature of DMH1-treated fish $(/)$ is irregular. No Zns5-positive cells are observed. The arrows indicate the width of the wound epidermis (w-ep), which is thickened in DMH1-treated fins $(n=5)$. 


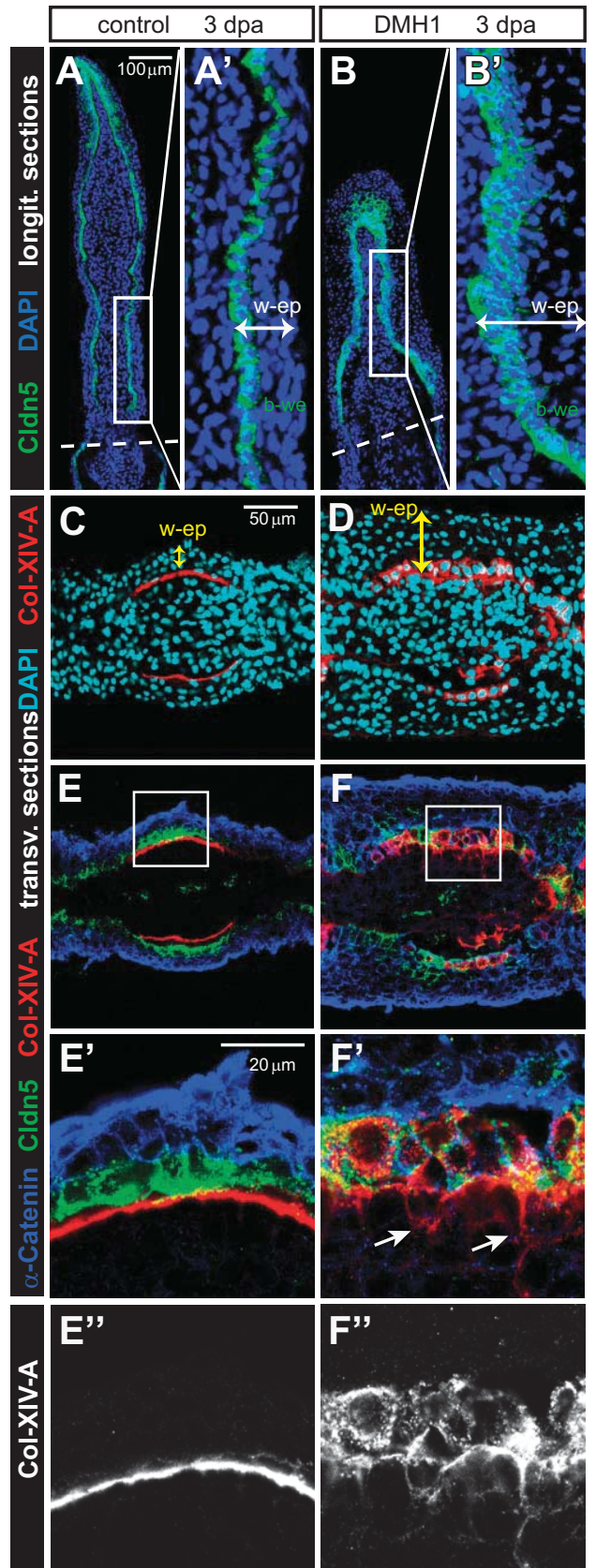

Figure 5. BMP signaling is required for the organization of the basal epithelium of the regenerating rays. $A$ and $B$ ) Longitudinal (longit.) sections of the fins at $3 \mathrm{dpa}$, immunostained against a tightjunction marker, Cldn5 (green). $A$ and $A^{\prime}$ ) In control fins, the basal wound epithelium (b-we) forms a Cldn5-labeled monolayer. $B$ and $\left.B^{\prime}\right)$ DMH1-treated fins develop a multilayered disorganized basal epithelium (b-we) that expresses Cldn5. The wound epidermis (w-ep) is twice thicker in DMH1-treated fish $(\mathrm{n}=5) . G-F)$ Transversal (transv.) sections of the fin outgrowth at $3 \mathrm{dpa}$, immunostained against an adherens-junction marker ( $\alpha$-Catenin, blue), Cldn 5 (green), ColXIV-A (red), and DAPI (cyan). $E, F, E^{\prime}, F^{\prime}, E^{\prime \prime}$, and $F^{\prime \prime}$ ) ColXIV-A forms a BM below the Cldn5-positive epithelium in control $\left(E-E^{\prime \prime}\right)$, but not in DMH1-treated fins $\left(F-F^{\prime \prime}\right)$, in which it displays an intracellular localization or a disorganized extracellular distribution (arrows).

$\mathbf{5} \boldsymbol{A}-\boldsymbol{D})$. To examine the role of BMP signaling in the organization of the wound epithelium, we highlighted the basal epithelium using immunofluorescence staining against
Cldn5, which is a component of tight junctions (34). In control fins at $3 \mathrm{dpa}$, Cldn5 labeled a single row of cells of the basal epithelium in the lateral part of the outgrowth (Fig. 5A). In DMH1-treated fins, Cldn5-positive cells lost their aligned organization, accumulating within 2-3 cell layers (Fig. $5 B$ ). Thus, BMP signaling is required to organize the basal epithelium.

To determine the cellular causes of this phenotype, we analyzed a component of the basement membrane (BM), ColXIV-A, which is specific for embryonic but not mature epithelia in zebrafish (35). In addition, we visualized the basal layer with a tight-junction marker, Cldn5, whereas the spinous layer was labeled with an adherens-junction marker, $\alpha$-Catenin (Fig. $5 E, F$ ). In control fish, the Cldn5-expressing epithelium was underlined by the ColXIV-A layer (Fig. $5 E-E^{\prime \prime}$ ). By contrast, such a structure was not deposited in DMH1treated fins (Fig. $5 F-F^{\prime}$ ). Instead, ColXIV-A was retained in the cytoplasm of the Cldn5-expressing epithelium. We concluded that BMP signaling is essential for the establishment of the specialized $\mathrm{BM}$ of the regenerating rays.

To further investigate the molecular causes associated with the structural abnormalities of the wound epidermis, we analyzed the expression of several genes encoding signaling-related proteins. First, we tested the expression of $w n t 5 b$, which is an important signaling molecule expressed in the blastema and in the basal wound epithelium (17). DMH1 treatment suppressed the transcription of this gene in the epidermis, without affecting its expression in the blastema (Fig. 6A-F). Thus, BMP regulates wnt5b expression specifically in the wound epithelium. Then, we tested the expression of lef1 and shha, which are normally confined to the subapical domain of the wound epithelium (26, 36). We found that the inhibition of BMP signaling did not affect lef1 and shha expression, suggesting that neither of these genes is a BMP target (Fig. 6G-J) We concluded that BMP signaling may, at least partially, control the organization of the wound epidermis through the activation of $w n t 5 b$ expression, an instructive factor during fin regeneration.

\section{Inhibition of BMP signaling impairs actinotrichia formation}

Actinotrichia are skeletal spikes composed of actinodin and collagen proteins that support the distal margin of the fin $(18,37)$. The mechanisms regulating actinotrichia regeneration in adult fin are unknown. We developed antibodies against And1, which is an essential component of these skeletal structures $(18,37)$. At 3 dpa, we detected actinotrichial fibers between the wound epidermis and the blastema (Fig. 7A). We found that the DMH1 treatment repressed deposition of And1-positive fibers, suggesting that BMP signaling is essential for actinotrichia formation (Fig. $7 B$ ). To determine whether BMP regulates the transcription of and1 gene, we performed in situ hybridization. At 3 dpa, and 1 transcript was detected in the blastema and in the basal layer of the wound epidermis (Fig. $7 C, E$ ). In DMH1-treated fins, and1 was expressed only in the blastema, but it was nearly not detectable in wound epidermis (Fig. $7 D, F)$. This situation is reminiscent of the results obtained for the $w n t 5 b$ gene, in which solely the epidermal expression but not the blastemal expression was affected by the inhibition of BMP signaling (Fig. $6 A-F$ ). Thus, our 
Figure 6. $w n t 5 b$ is a target of BMP signaling in the wound epithelium, but not in the blastema. $A-F)$ In situ hybridization of longitudinal $(A, B, E$, and $F)$ and transversal $(C$ and $D)$ fin sections using wnt $5 b$ antisense probe. $A, C$, and $E$ ) In control fins, wnt5b is expressed in the basal wound epidermis and the distal blastema. $B, D$, and $F$ ) In DMH1-treated fins, $w n t 5 b$ is detected only in the blastema, but not in the wound epidermis. $F$ ) One day of DMH1 treatment is sufficient to suppress $w n t 5 b$ in wound epidermis. Dashed red lines demarcate the borders of the basal wound epithelium (b-we) $(n>5) . G-J)$ lef1 and shha are not targets of BMP signaling during fin regeneration. Similarly to control, DMH1-treated fins express both genes in the basal wound epithelium (b-we) $(n>4)$.

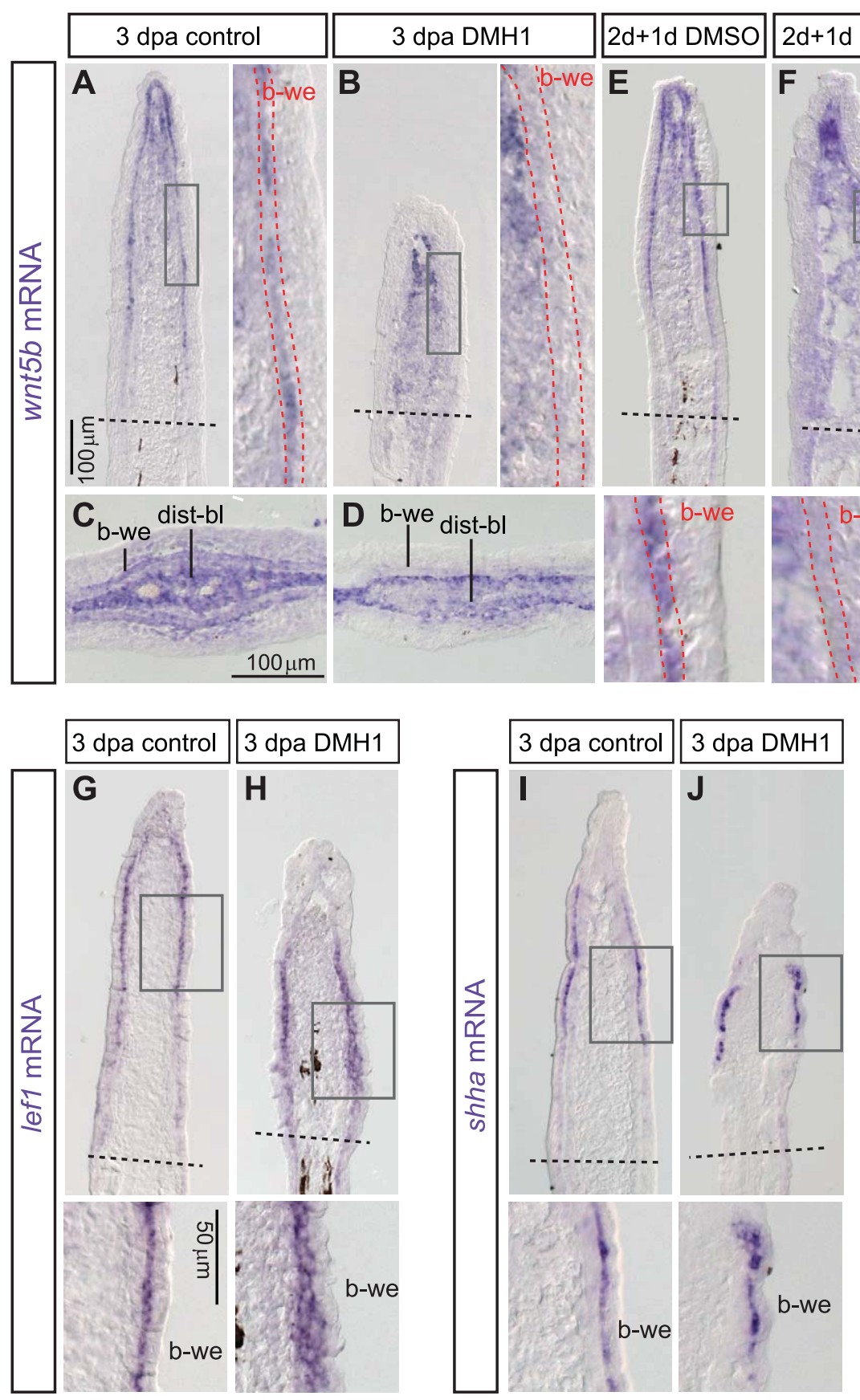

analysis revealed that the BMP-dependent and1 expression in the wound epidermis is critical for actinotrichia formation. Taken together, BMP signaling induces the epithelial transcription of 2 genes encoding the signaling molecule $w n t 5 b$ and the structural protein and1.

To test whether the lack of actinotrichia is a primary or secondary effect of blocking BMP signaling, we performed a pulse-treatment experiment, in which fish were exposed to DMH1 for only $1 \mathrm{~d}$, starting at $2 \mathrm{dpa}$. To determine the And1 distribution relative to the bones, fins were immunostained for Zns5, which is a cell surface pan-osteoblast marker. The actinotrichial network starts to be established at $2 \mathrm{dpa}$, and it doubles its size over the course of the next day (Fig. $7 G, H$ ). Treatment with DMH1 during d 3 of regeneration blocked expansion of the And1-labeled fibers, resulting in a pattern similar to the one observed before the treatment at 2 dpa (Fig. 7I). Consistently, the levels of and1 transcription were down-regulated by the pulse treatment with DMH1, as shown by quantitative RT-PCR and in situ hybridization (Fig. 7J-L). This demonstrates that BMP signaling regulates de novo formation of the actinotrichia by inducing and1 expression in the wound epidermis.

\section{DISCUSSION}

$\mathrm{BMP}$ is one of the multitasking pathways that can regulate diverse cellular decisions in a context-dependent manner $(38,39)$. The canonical signaling from diverse BMP ligands and receptors merges at the level of $\mathrm{pSmad} 1 / 5 / 8$, which 

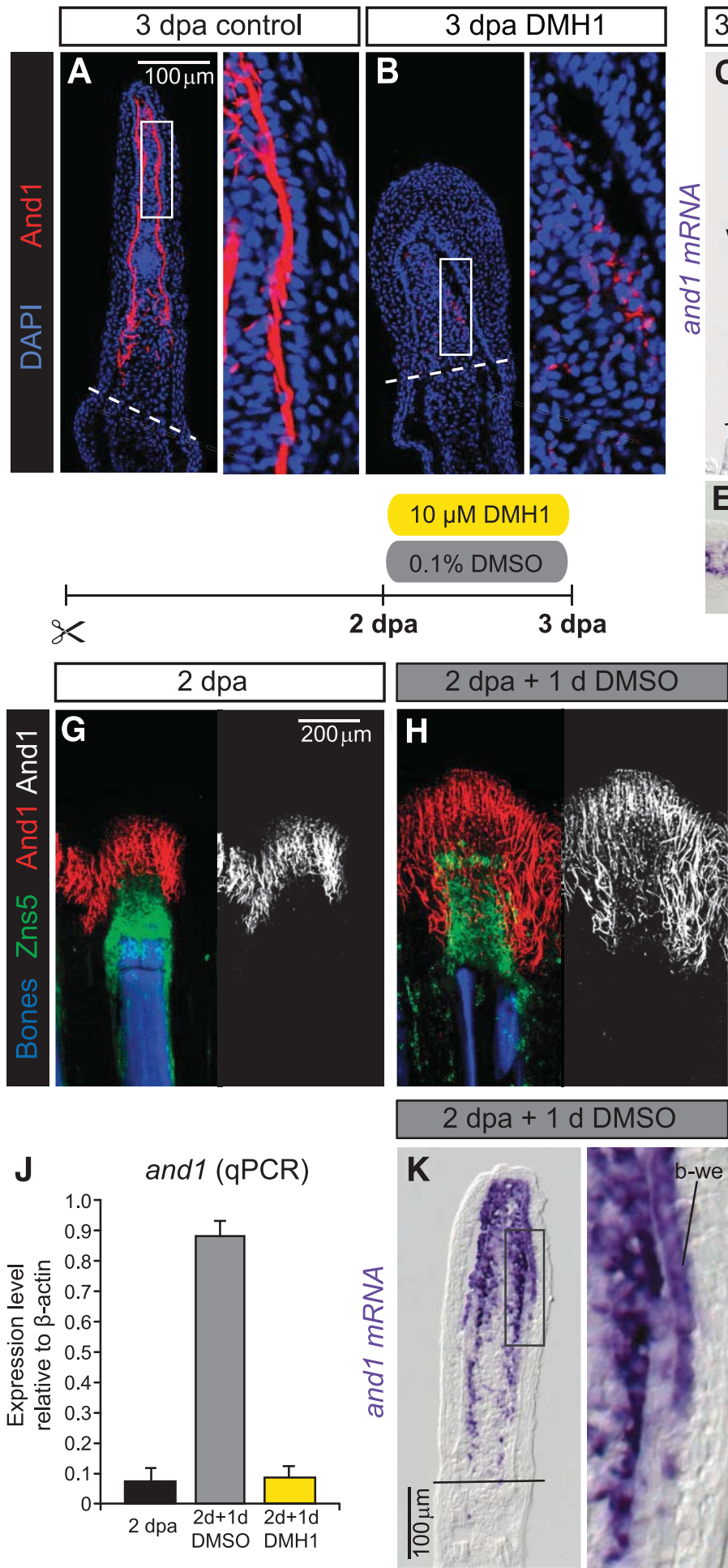
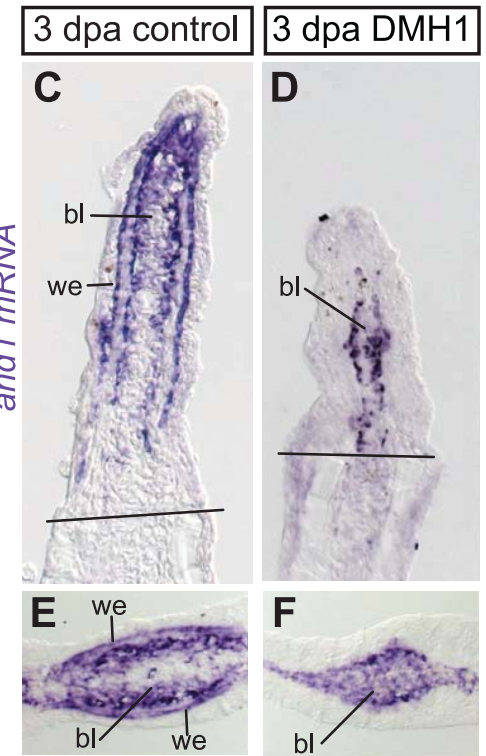

D

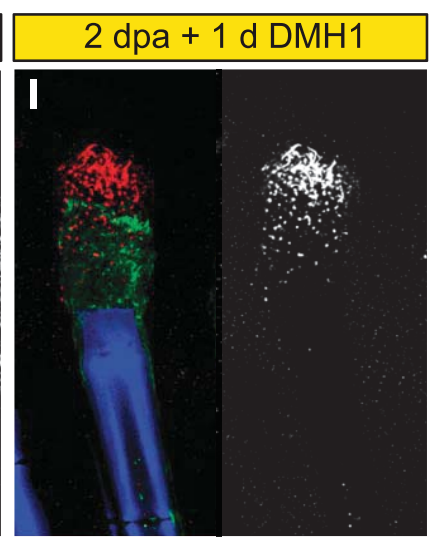

2 dpa + 1 d DMH1

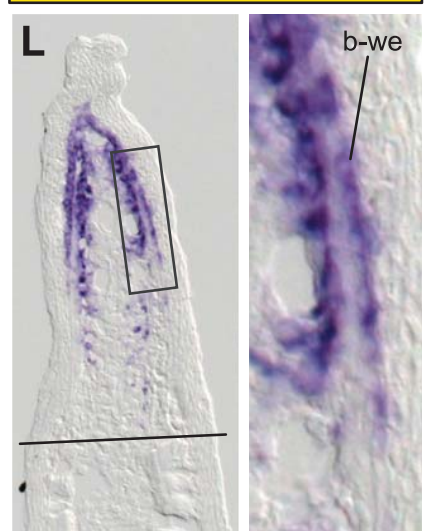

Figure 7. BMP signaling is required for actinotrichia formation. $A$ and $B$ ) Immunofluorescence staining of fin sections at 3 dpa. A) Control fins contain And1positive actinotrichial fibers (red) between the wound epidermis and the blastema. B) DMH1-treated fins lack the actinotrichia; only a few remnant fibers are detected in the blastema $(n=5) . C-F)$ In situ hybridization of fin longitudinal $(C$ and $D)$ and transversal $(E$ and $F$ ) sections at $3 \mathrm{dpa}$ using and 1 antisense probe. $C$ and $E$ ) Control fins express and1 in the basal layer of wound epidermis (we) and in the blastema (bl). $D$ and $F$ ) The DMH1 treatment suppressed and1 transcription in wound epidermis, but it did not affect and 1 expression in the blastema $(n=3 / 5) . G-I)$ The tip of a ray of whole-mount fins after $1 \mathrm{~d}$ of DMH1 treatment starting at 2 dpa (graph with experimental design) was immunostained against Andl (red) and the osteoblast marker Zns5 (green). The bone matrix is shown in blue (autofluorescence). $G$ At 2 dpa, actinotrichia fibers (red) start to form in the outgrowth. $H$ ) At 3 dpa, control fins display enhanced And1 expression. I) A pulse DMH1 treatment interrupts deposition of new actinotrichial structures $(n=5) . J)$ Quantitative RT-PCR analysis demonstrates that $1 \mathrm{~d}$ of DMH1 treatment starting at $2 \mathrm{dpa}$ is sufficient to suppress and 1 transcription. Normalized gene expression was calculated relative to ubiquitously expressed $\beta$-actin with the value $1 \quad(n=3$ samples; each was prepared from 8 fins). $K$ and $L$ ) In situ hybridization of fin sections displays reduced and1 expression after $1 \mathrm{~d}$ of DMH1 treatment starting at 2 dpa $(n>3)$.

can directly induce target gene expression, such as $i d 1$. Here, we focused on $i d 1$ transcription to establish for the first time a map of the BMP signaling activity in the regenerating fin. We found the BMP readout in a large variety of structures, such as distal mesenchyme, osteoblasts, arteries, and wound epidermis. To study the function of this pathway, we blocked the BMP type I receptors with a selective chemical inhibitor, DMH1 $(27,28,40)$. This approach resulted in an arrest of the regenerative process shortly after the initiation phase. Using a combination of transgenic, immunofluorescence, histologic, and in situ hybridization techniques, we identified novel functions of BMP during regenerative angiogenesis, regulation of the wound epithelium, and actinotrichia formation. Based on the BMP activity profile and its functional relevance, we propose a model about the regenerative processes 
occurring during the postblastema formation phase of fin regeneration. We identified that even a prolonged DMH1 treatment for 3 dpa did not affect blastema formation, but it abruptly arrested progression of regeneration. This excludes BMP from the network of signaling pathways required for the initiation of the regenerative programs, such as Activin- $\beta A$, FGF, and retinoic acid $(3,12)$. Our data support the notion that BMP acts on the cells that have been recruited into the outgrowth to promote morphogenesis of multiple fin structures, including bones, wound epidermis, blood vessels, and actinotrichia. Our findings are consistent with the general developmental concepts about the multifunctional feature of the BMP signaling pathway.

Although $i d 1$ promoter has been extensively studied to identify the BMP-responsive element, the vertebrate genome comprises several other Id-related proteins that are less characterized at the biochemical level. In this study, we analyzed the expression pattern of $3 i d$ homologs, $i d 1, i d 2 a$, and $i d 3$, at $3 \mathrm{dpa}$, when the DMH1-induced phenotype becomes evident. In situ hybridization data revealed a partially overlapping distribution of all these $i d$ genes in the distal blastema, wound epidermis, and osteoblasts. In addition, $i d 1$ and $i d 3$ were expressed in the regenerating blood vessels of the outgrowth. Our study revealed that only $i d 1$ and $i d 3$ are targets of BMP signaling because the DMH1 treatment completely suppressed their expression, whereas it did not prevent id2a expression. Interestingly, an overlapping expression pattern of $i d 1$ and $i d 3$ genes has been also reported during mammalian embryogenesis and tumorigenesis (41), and knockout mouse studies demonstrated a redundancy between these genes (42). Thus, the close transcriptional relation between $i d 1$ and $i d 3$ genes appears to be conserved among vertebrates.

We have shown that BMP activity induces $i d 1 / 3$ transcription in multiple tissues, suggesting a variety of functions during fin regeneration. We systematically examined the effect of DMH1 treatment on regeneration of $4 \mathrm{id} 1 / 3$ expressing tissues. First, $i d$ genes were induced in the distalmost blastema that comprises slowly proliferating mesenchymal progenitors. This induction might be dependent on $b m p 4$, which is expressed at the tip of the blastema (15), and/or on $b m p 7 a$, which we identified in this study. In mammals, the expression of $i d$ genes is low in differentiated adult tissues, but it becomes abundant in proliferating embryonic cells, adult stem cells, and tumors (41). Although in mammalian systems, BMP4-induced id expression was reported to preserve stem cell identity and to prevent premature differentiation (43), we did not observe an analogic outcome in the fibroblast progenitors in the blastema. The inhibition of BMP signaling neither severely affected blastema cell proliferation nor the expression of several blastema markers, such as Tenascin C, aldh1a2, and $m s x B$. It is possible that the BMP-independent $i d 2 a$ expression in the mesenchyme of the outgrowth was sufficient to compensate for the absence of $i d 1 / 3$.

In contrast to mesenchyme, several other fin tissues displayed a phenotype after inhibition of BMP signaling. We identified the BMP-dependent expression of $i d 1 / 3$ genes in osteoblasts. We used a set of transgenic fish to support the notion that BMP signaling guides the activated bone progenitor cells through the osteogenic differentiation program, which is consistent with previous studies
(15-17). Besides the bones, we identified previously unrecognized abnormalities in wound epidermis, actinotrichia, and blood vessels (Fig. 8).

We reported that the inhibition of BMP resulted in the thickening and malformation of the wound epidermis. By using a cell tight-junction marker, Cldn5, we identified a disorganization of the basal epithelium in the regenerating rays. The abnormal alignment of the basal epithelium was associated with a defective BM, as visualized by the distribution of ColXIV-A. The inhibition of BMP did not prevent the expression of ColXIV-A, but it blocked its secretion and deposition into the BM. The mechanisms underlying this phenotype require further investigations. ColXIV-A has been shown to be required for the proper function of embryonic BMs in undifferentiated zebrafish epithelia (35). It is reasonable to expect that the defects in the composition of the BM might be a direct cause of the misaligned wound epithelium and, subsequently, thickened epidermis (Fig. 8A).

We found that the defective wound epithelium displayed normal expression of 2 wound epithelial markers, shha and lef1, indicating that these genes are not targets of BMP signaling. However, we identified that DMH1 treatment inhibited the expression of 2 other genes in the wound epithelium, namely wnt5b and and1, without affecting their expression in the blastema. This finding suggests the existence of differential tissue-dependent mechanisms that induce wnt $5 b$ and and 1 in both tissues of the fin outgrowth. Thus, we identified a novel role of BMP signaling in the regulation of a subset of wound epithelial genes. The regulation of $w n t 5 b$ brings new insights about the network of signaling pathways that orchestrate growth and differentiation of the regenerating fin.

Actinotrichia are fish-specific extracellular fibers that are assembled at the epithelial-mesenchymal interface of the outgrowth to stabilize the fin regenerate $(37,44)$. The mechanisms that regulate actinotrichia formation are unknown. We generated a new antibody against And1, which reliably labels actinotrichia fibers. The DMH1-mediated inhibition of and1 in the wound epidermis was sufficient to abolish actinotrichia formation, even in the presence of and 1 transcripts in the blastema. Our data indicate that the wound epidermis plays an essential role for the formation of the actinotrichia (Fig. 8A).

Another novel and important aspect is represented by the role of BMP signaling during regenerative angiogenesis. Two findings suggest that BMP acts directly in the blood vessels of the fin outgrowth, namely the $\mathrm{pSmad} 1 / 5 / 8 \mathrm{im}$ munoreactivity and $i d 1 / i d 3$ gene expression in the endothelial cells. The angiogenesis during fin regeneration has been shown to be dependent on VEGF signaling (14). At $3 \mathrm{dpa}$, the vascular plexus is formed, which progressively becomes restructured into veins and arteries within the next week of regeneration (13). The inhibition of BMP signaling did not block the initiation of angiogenesis, but it impaired subsequent maturation of the recruited blood vessels, resulting in malformed and leaky capillaries (Fig. $8 B)$. Our data suggest that BMP signaling is required for remodeling of the plexus into distinguishable arteries and veins. Further studies are required to determine the downstream targets of the BMP activity that regulate the integrity and mobilization of the regenerating vasculature. Similarly, BMP signaling is known to be involved in angiogenesis in mammalian model systems (45). Interestingly, 


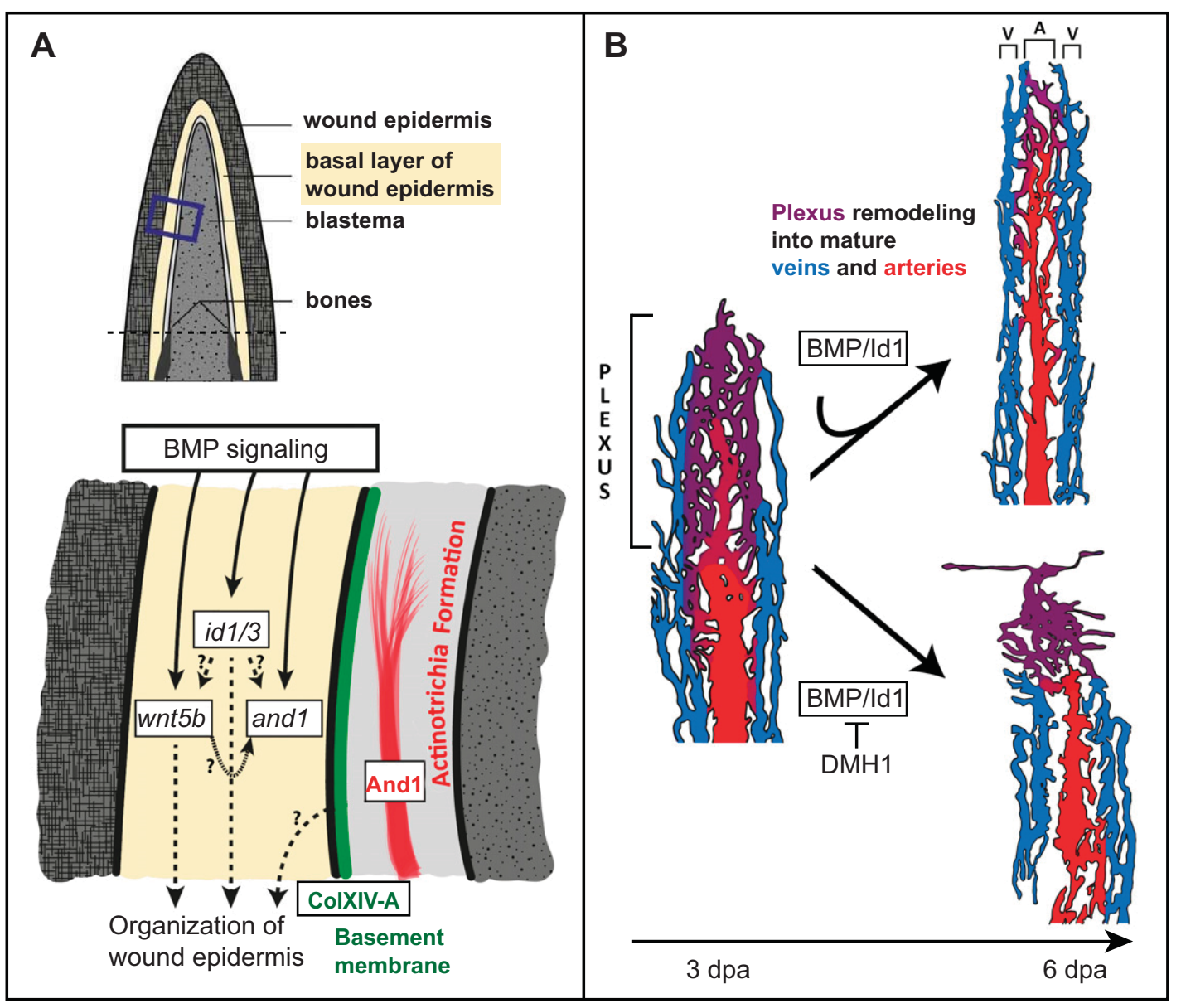

Figure 8. Model of the novel BMP activities in the wound epithelium and the vasculature of the fin regenerate. A) BMP induces $i d 1 / 3$, wnt $5 b$, and and1 expression in the basal wound epithelium. This signaling is required for the deposition of ColXIV-A into the $\mathrm{BM}$ of the regenerating rays and for actinotrichia formation between the wound epidermis and the blastema. B) BMP promotes vascular plexus remodeling into structured blood vessels in the fin outgrowth. A, artery; $\mathrm{V}$, vein.

id1/id3 double-knockout mice develop a normal vascular system with regard to the main blood vessels, but the capillaries are dilated and malformed leading to hemorrhages, particularly in the brain, causing lethality (46). During tumorigenesis, $i d 1 / i d 3$ are not required for the initial phase of angiogenesis but for the progressive maturation and invasion of blood vessel into the growing tissue mass (41). It is important to recognize the parallels between the remodeling of vascular plexus during tumor and blastema angiogenesis. In both contexts, $i d 1 / i d 3$ might have a similar function in remodeling of blood vessels during the rapid growth of tissue mass in adult vertebrates. Thus, the maturation and structuring of the vascular network from rapidly growing capillaries might depend on similar molecular circuits in diverse vertebrates. Based on our study, the regenerative angiogenesis of the fin might contribute a valuable in vivo model system for medical research of tumor angiogenesis.

The authors thank V. Zimmermann for excellent technical assistance and for fish care; C. Pfefferli, B. Chassot, and A.-S. de Preux Charles for critical reading of the manuscript; M. Affolter, S. Seyfried, and G. Weidinger for providing transgenic fish; and F.R. for Collagen XIV-A antibody. This work was supported by the Swiss National Science Foundation (Grants 310030 159995 and CRSII3_147675).

\section{REFERENCES}

1. Simon, A., and Tanaka, E. M. (2013) Limb regeneration. Wiley Interdiscip. Rev. Dev. Biol. 2, 291-300

2. Yoshinari, N., and Kawakami, A. (2011) Mature and juvenile tissue models of regeneration in small fish species. Biol. Bull. 221, 62-78

3. Gemberling, M., Bailey, T. J., Hyde, D. R., and Poss, K. D. (2013) The zebrafish as a model for complex tissue regeneration. Trends Genet. 29, 611-620

4. Pfefferli, C., and Jaźwińska, A. (2015) The art of fin regeneration in zebrafish. Regeneration 2, 72-83

5. Tu, S., and Johnson, S. L. (2011) Fate restriction in the growing and regenerating zebrafish fin. Dev. Cell 20, 725-732

6. Knopf, F., Hammond, C., Chekuru, A., Kurth, T., Hans, S., Weber, C. W., Mahatma, G., Fisher, S., Brand, M., Schulte-Merker, S., and Weidinger, G. (2011) Bone regenerates via dedifferentiation of osteoblasts in the zebrafish fin. Dev. Cell 20, 713-724

7. Sousa, S., Afonso, N., Bensimon-Brito, A., Fonseca, M., Simões, M. Leon, J., Roehl, H., Cancela, M. L., and Jacinto, A. (2011) Differentiated skeletal cells contribute to blastema formation during zebrafish fin regeneration. Development 138, 3897-3905

8. Stewart, S., and Stankunas, K. (2012) Limited dedifferentiation provides replacement tissue during zebrafish fin regeneration. Dev. Biol. 365, 339-349

9. Tornini, V.A., and Poss, K. D. (2014) Keeping at arm's length during regeneration. Dev. Cell 29, 139-145

10. Jopling, C., Boue, S., and Izpisua Belmonte, J. C. (2011) Dedifferentiation, transdifferentiation and reprogramming: three routes to regeneration. Nat. Rev. Mol. Cell Biol. 12, 79-89

11. Brockes, J. P., and Kumar, A. (2008) Comparative aspects of animal regeneration. Annu. Rev. Cell Dev. Biol. 24, 525-549 
12. Wehner, D., and Weidinger, G. (2015) Signaling networks organizing regenerative growth of the zebrafish fin. Trends Genet. 31, 336-343

13. Huang, C. C., Lawson, N. D., Weinstein, B. M., and Johnson, S. L. (2003) reg6 is required for branching morphogenesis during blood vessel regeneration in zebrafish caudal fins. Dev. Biol. 264, 263-274

14. Bayliss, P. E., Bellavance, K. L., Whitehead, G. G., Abrams, J. M., Aegerter, S., Robbins, H. S., Cowan, D. B., Keating, M. T., O'Reilly, T., Wood, J. M., Roberts, T. M., and Chan, J. (2006) Chemical modulation of receptor signaling inhibits regenerative angiogenesis in adult zebrafish. Nat. Chem. Biol. 2, 265-273

15. Smith, A., Avaron, F., Guay, D., Padhi, B. K., and Akimenko, M. A (2006) Inhibition of BMP signaling during zebrafish fin regeneration disrupts fin growth and scleroblasts differentiation and function. Dev. Biol. 299, 438-454

16. Quint, E., Smith, A., Avaron, F., Laforest, L., Miles, J., Gaffield, W., and Akimenko, M. A. (2002) Bone patterning is altered in the regenerating zebrafish caudal fin after ectopic expression of sonic hedgehog and bmp2b or exposure to cyclopamine. Proc. Natl. Acad. Sci. USA 99, 8713-8718

17. Stewart, S., Gomez, A. W., Armstrong, B. E., Henner, A., and Stankunas, K. (2014) Sequential and opposing activities of Wnt and BMP coordinate zebrafish bone regeneration. Cell Reports 6, 482-498

18. Durán, I., Marí-Beffa, M., Santamaría, J. A., Becerra, J., and Santos-Ruiz, L. (2011) Actinotrichia collagens and their role in fin formation. Dev. Biol. 354, 160-172

19. Miyazono, K., Kamiya, Y., and Morikawa, M. (2010) Bone morphogenetic protein receptors and signal transduction. J. Biochem. 147, 35-51

20. Jin, S. W., Beis, D., Mitchell, T., Chen, J. N., and Stainier, D. Y. (2005) Cellular and molecular analyses of vascular tube and lumen formation in zebrafish. Development 132, 5199-5209

21. Blum, Y., Belting, H. G., Ellertsdottir, E., Herwig, L., Lüders, F., and Affolter, M. (2008) Complex cell rearrangements during intersegmental vessel sprouting and vessel fusion in the zebrafish embryo. Dev. Biol. 316, 312-322

22. Motoike, T., Loughna, S., Perens, E., Roman, B. L., Liao, W., Chau, T. C., Richardson, C. D., Kawate, T., Kuno, J., Weinstein, B. M., Stainier, D. Y., and Sato, T. N. (2000) Universal GFP reporter for the study of vascular development. Genesis 28, 75-81

23. Chocron, S., Verhoeven, M. C., Rentzsch, F., Hammerschmidt, M., and Bakkers, J. (2007) Zebrafish Bmp4 regulates left-right asymmetry at two distinct developmental time points. Dev. Biol. 305, 577-588

24. Chablais, F., and Jazwinska, A. (2010) IGF signaling between blastema and wound epidermis is required for fin regeneration. Development 137, 871-879

25. Jaźwińska, A., Badakov, R., and Keating, M. T. (2007) Activin-betaA signaling is required for zebrafish fin regeneration. Curr. Biol. 17 , 1390-1395

26. Laforest, L., Brown, C. W., Poleo, G., Géraudie, J., Tada, M., Ekker, M., and Akimenko, M. A. (1998) Involvement of the sonic hedgehog, patched 1 and bmp2 genes in patterning of the zebrafish dermal fin ravs. Development 125, 4175-4184

27. Engers, D. W., Frist, A. Y., Lindsley, C. W., Hong, C. C., and Hopkins, C. R. (2013) Synthesis and structure-activity relationships of a nove and selective bone morphogenetic protein receptor (BMP) inhibitor derived from the pyrazolo[1.5-a]pyrimidine scaffold of dorsomorphin: the discovery of ML347 as an ALK2 versus ALK3 selective MLPCN probe. Bioorg. Med. Chem. Lett. 23, 3248-3252

28. Hao, J., Ho, J. N., Lewis, J. A., Karim, K. A., Daniels, R. N., Gentry, P. R., Hopkins, C. R., Lindsley, C. W., and Hong, C. C. (2010) In vivo structure-activity relationship study of dorsomorphin analogues identifies selective VEGF and BMP inhibitors. ACS Chem. Biol. 5, 245-253

29. Korchynskyi, O., and ten Dijke, P. (2002) Identification and functional characterization of distinct critically important bone morphogenetic protein-specific response elements in the Id1 promoter. J. Biol. Chem. 277, 4883-4891

30. Nechiporuk, A., and Keating, M. T. (2002) A proliferation gradient between proximal and msxb-expressing distal blastema directs zebrafish fin regeneration. Development 129, 2607-2617

31. Mathew, L. K., Sengupta, S., Franzosa, J. A., Perry, J., La Du, J., Andreasen, E. A., and Tanguay, R. L. (2009) Comparative expression profiling reveals an essential role for raldh2 in epimorphic regeneration. J. Biol. Chem. 284, 33642-33653

32. Poss, K. D., Keating, M. T., and Nechiporuk, A. (2003) Tales of regeneration in zebrafish. Dev. Dyn. 226, 202-210

33. Whitehead, G. G., Makino, S., Lien, C. L., and Keating, M. T. (2005) fgf20 is essential for initiating zebrafish fin regeneration. Science 310, 1957-1960

34. Van Itallie, C. M., and Anderson, J. M. (2013) Claudin interactions in and out of the tight junction. Tissue Barriers 1, e25247

35. Bader, H. L., Lambert, E., Guiraud, A., Malbouyres, M., Driever, W., Koch, M., and Ruggiero, F. (2013) Zebrafish collagen XIV is transiently expressed in epithelia and is required for proper function of certain basement membranes. J. Biol. Chem. 288, 6777-6787

36. Poss, K. D., Shen, J., and Keating, M. T. (2000) Induction of lef during zebrafish fin regeneration. Dev. Dyn. 219, 282-286

37. Zhang, J., Wagh, P., Guay, D., Sanchez-Pulido, L., Padhi, B. K., Korzh, V., Andrade-Navarro, M. A., and Akimenko, M. A. (2010) Loss of fish actinotrichia proteins and the fin-to-limb transition. Nature 466 , 234-237

38. Miyazono, K., ten Dijke, P., and Heldin, C. H. (2000) TGF-beta signaling by Smad proteins. Adv. Immunol. 75, 115-157

39. Wagner, D. O., Sieber, C., Bhushan, R., Börgermann, J. H., Graf, D. and Knaus, P. (2010) BMPs: from bone to body morphogenetic proteins. Sci. Signal. 3, mr1

40. Laux, D. W., Febbo, J. A., and Roman, B. L. (2011) Dynamic analysis of BMP-responsive smad activity in live zebrafish embryos. Dev. Dyn. 240, 682-694

41. Lasorella, A., Benezra, R., and Iavarone, A. (2014) The ID proteins: master regulators of cancer stem cells and tumour aggressiveness. Nat. Rev. Cancer 14, 77-91

42. ten Dijke, P., Korchynskyi, O., Valdimarsdottir, G., and Goumans, M. J. (2003) Controlling cell fate by bone morphogenetic protein receptors. Mol. Cell. Endocrinol. 211, 105-113

43. Ying, Q. L., Nichols, J., Chambers, I., and Smith, A. (2003) BMP induction of Id proteins suppresses differentiation and sustains embryonic stem cell self-renewal in collaboration with STAT3. Cell 115, 281-292

44. Pfefferli, C., Müller, F., Jaźwińska, A., and Wicky, C. (2014) Specific $\mathrm{NuRD}$ components are required for fin regeneration in zebrafish. BMC Biol. 12, 30

45. Cai, J., Pardali, E., Sánchez-Duffhues, G., and ten Dijke, P. (2012) BMP signaling in vascular diseases. FEBS Lett. 586, 1993-2002

46. Lyden, D., Young, A. Z., Zagzag, D., Yan, W., Gerald, W., O'Reilly, R., Bader, B. L., Hynes, R. O., Zhuang, Y., Manova, K., and Benezra, R. (1999) Id1 and Id3 are required for neurogenesis, angiogenesis and vascularization of tumour xenografts. Nature 401, 670-677 